Übersichten

Monatsschr Kinderheilkd 2020 · 168:615-627 https://doi.org/10.1007/s00112-020-00919-7 Online publiziert: 21. April 2020

(C) Der/die Autor(en) 2020

\section{Redaktion}

B. Koletzko, München

T. Lücke, Bochum

E. Mayatepek, Düsseldorf

N. Wagner, Aachen

S. Wirth, Wuppertal

F. Zepp, Mainz

\author{
A. Streng' $\cdot$ K. Hartmann' $\cdot$ J. Armann² $\cdot$ R. Berner' ${ }^{2} \cdot$ J. G. Liese' \\ ${ }^{1}$ Kinderklinik und Poliklinik, Universitätsklinikum an der Julius-Maximilians-Universität Würzburg, \\ Würzburg, Deutschland \\ ${ }^{2}$ Klinik und Poliklinik für Kinder- und Jugendmedizin, Universitätsklinikum Carl Gustav Carus an der \\ Technischen Universität Dresden, Dresden, Deutschland
}

\title{
COVID-19 bei hospitalisierten Kindern und Jugendlichen
}

\section{Ein systematischer Review zu publizierten Fallserien (Stand 31.03.2020) und erste Daten aus Deutschland}

\section{Einleitung}

\section{Bei Kindern scheinen COVID-19- Erkrankungen durch SARS-CoV-2 insgesamt leichter zu verlaufen als bei Erwachsenen. Bislang gibt es nur wenige Veröffentlichungen zu klinischem Verlauf, Komplikatio- nen und Therapie bei Kindern. Der vorliegende systematische Review zu Fallserien bei hospitalisierten Kindern gibt einen Überblick zum aktuellen Stand der wissenschaft- lichen Evidenz, inklusive erster Daten aus Deutschland aus einem Krankenhaus-Survey der Deut- schen Gesellschaft für Pädiatrische Infektiologie e. V. (DGPI).}

Das neue Coronavirus SARS-CoV-2 hat sich innerhalb weniger Wochen seit seinem ersten bekannten Auftreten in Wuhan/Hubei/China im Dezember 2019 in nahezu alle Länder verbreitet. Am 11.03.2020 deklarierte die WHO den Ausbruch als Pandemie. Laut dem European Centre for Disease Prevention and Control (ECDC) wurden mit dem Stand 02.04.2020 weltweit insgesamt 928.437 durch das Virus verursachte Erkrankungen (,coronavirus disease 2019“, COVID-19) bestätigt, davon waren 46.891 Patienten verstorben $(5,1 \%)$ [11]. In Deutschland waren bis zu diesem Zeitpunkt 73.522 Fälle gemeldet, darunter 872 Todesfälle (1,2\%).
An COVID-19 erkranken zumeist erwachsene Patienten ( $\geq 95 \%)$. Nach der aktuellen ECDC-Surveillance sind Fieber $(47 \%)$, trockener/produktiver Husten (25\%), Halsschmerzen (16\%), Abgeschlagenheit (6\%) und Schmerzen (5\%) die häufigsten Symptome [11]. Pulmonale Komplikationen, wie Bronchitis, Pneumonie und ARDS (,acute respiratory distress syndrome") sind vorwiegend bei Personen über 60 Jahre und Personen mit Grunderkrankungen beschrieben, darunter Patienten mit chronischen Lungen- und kardiovaskulären Erkrankungen, Hypertonie, Diabetes, kardialen und onkologischen Erkrankungen. Ca. $30 \%$ der bislang bekannten COVID-19Fälle in Europa wurden hospitalisiert. Bei ca. 4-14\% der Patienten nimmt die Krankheit einen schweren Verlauf; der Fall-Verstorbenen-Anteil (englisch „case-fatality rate“) in Europa liegt zwischen $0-9 \%$ und in China bei 2,3\% [11, 31]. Die häufigsten Komplikationen bei verstorbenen Patienten waren respiratorische Insuffizienz/ARDS (97-100\%), akutes Nierenversagen (25-29\%), akute Myokardschädigungen (10-77\%) und bakterielle Superinfektionen/septischer Schock (9-100\%) [5, 11].

Der erste pädiatrische Nachweis einer SARS-CoV-2-Infektion erfolgte in China am 20.01.2020 bei einem 10-jährigen asymptomatischen Kind im Rahmen einer Untersuchung von familiärer Transmission [4]. Danach wurden zum klinischen Verlauf von hospitalisierten Kindern mit COVID-19-Infektion in rascher Folge Einzelfallberichte und Fallserien unterschiedlicher Größe v.a. aus China publiziert.

Insgesamt scheint das Erkrankungsbild von COVID-19 bei Kindern einen überwiegend leichten Verlauf zu zeigen $[3,22,36]$, auch wenn vereinzelt Komplikationen besonders bei Patienten mit Risikofaktoren, wie z. B. kardialen, pulmonalen und immunologischen Grunderkrankungen, sowie bei Säuglingen berichtet wurden. Das Ziel des vorliegenden systematischen Reviews ist es, dem klinisch tätigen Pädiater eine Übersicht über den klinischen Verlauf, Komplikationen, Therapie und Prognose von COVID-19-Infektionen bei hospitalisierten Kindern anhand der bisher publizierten wissenschaftlichen Evidenz zu geben.

\section{Methodik}

Es wurde eine Literatursuche in $P u b$ Med zu den Stichworten ",child/children/ childhood/p(a)ediatric/p(a)ediatrician/ pregnancy/neonate/baby/infant/adolescent" AND „,corona/coronavirus/CoV/ nCoV/CoV2/SARS-CoV-2/Covid/ COVID/COVID-19“AND „2020“ durchgeführt (zuletzt am 31.03.2020). Referenzen relevanter Publikationen und Review-Artikel wurden manuell auf weitere relevante Artikel überprüft. Artikel wurden nur bei Vorliegen zumindest einer 


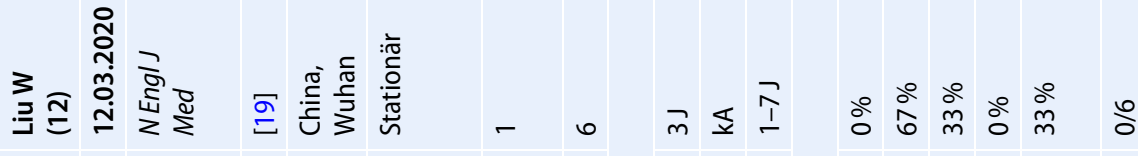

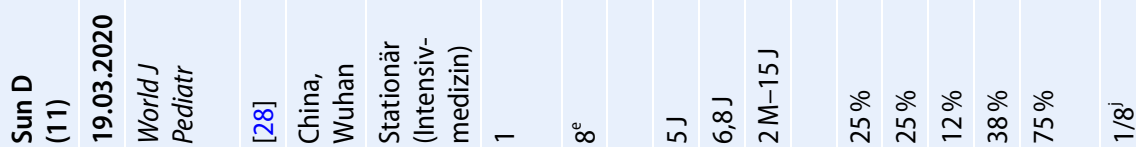

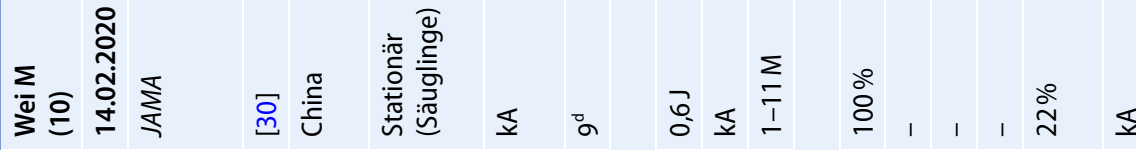

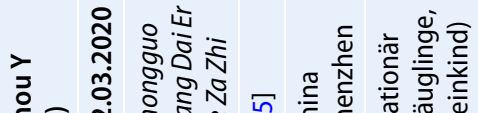

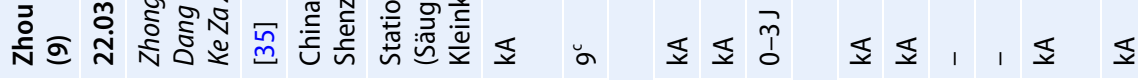

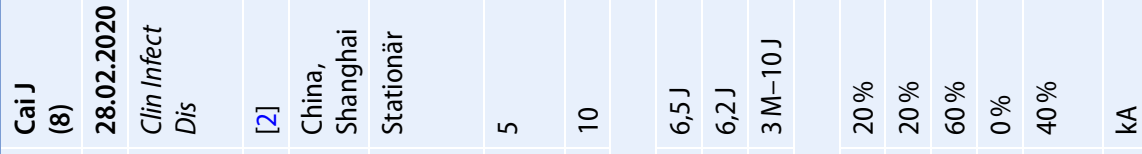

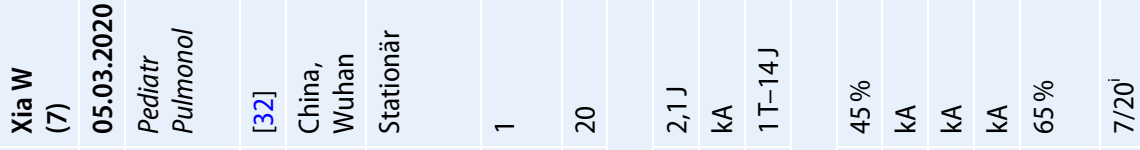

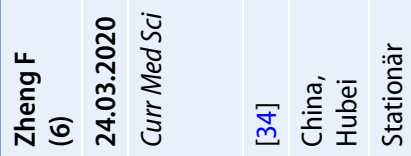

는

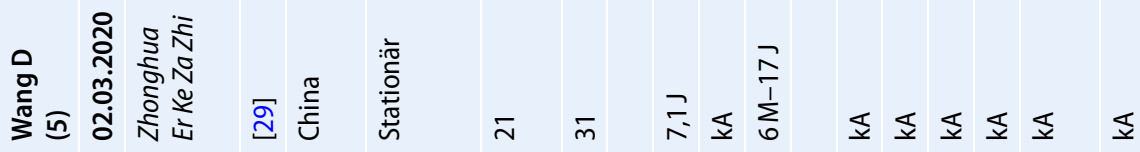

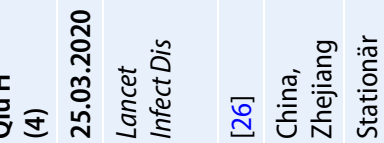

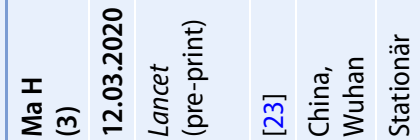

ֻุ广

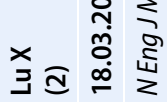

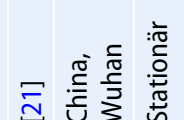
Nan

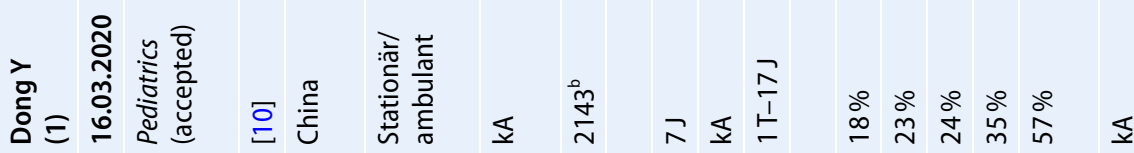


Monatsschr Kinderheilkd 2020 - 168:615-627 https://doi.org/10.1007/s00112-020-00919-7

(c) Der/die Autor(en) 2020

\section{A. Streng · K. Hartmann · J. Armann · R. Berner · J. G. Liese}

\section{COVID-19 bei hospitalisierten Kindern und Jugendlichen. Ein systematischer Review zu publizierten Fallserien (Stand 31.03.2020) und erste Daten aus Deutschland}

\section{Zusammenfassung}

Hintergrund. Das klinische Wissen zu Verlauf, Komplikationen und Therapie von COVID-

19 bei Kindern und Jugendlichen ist bisher begrenzt.

Ziel. Der vorliegende systematische Review fasst die aktuelle wissenschaftliche Evidenz zum klinischen Verlauf von COVID-19 bei hospitalisierten Kindern zusammen. Zusätzlich werden die ersten Daten aus Deutschland aus einem Kinderklinik-Survey der Deutschen Gesellschaft für Pädiatrische Infektiologie e.V. (DGPI) vorgestellt.

Methodik. Ausgewertet wurden insgesamt 12 Fallserien aus China mit 6 bis 2143 SARSCoV-2-infizierten Kindern, die über eine PubMed-Literatursuche bis zum 31.03.2020 identifiziert wurden. Die Datenbank des deutschlandweiten COVID-19 KinderklinikSurvey der DGPI wurde am 06.04.2020 abgefragt.

Ergebnisse. In den Fallserien lag das mediane Alter zwischen 2 und 7 Jahren, mit einem Anteil von Säuglingen von 18 bis $45 \%$. Am häufigsten berichtete Symptome waren Fieber und Husten; bei $40-100 \%$ lag eine radiologisch (zumeist $\mathrm{CT}$ ) bestätigte Lungenbeteiligung vor. Schwere/kritische Verläufe - inklusive 2 Todesfälle - wurden bei bis zu $8 \%$ der Kinder berichtet. Die Hospitalisierungsdauer lag bei 5 bis 20 Tagen. Der COVID-19-Survey der DGPI erfasste bis zum 06.04.2020 33 Kinder aus 21 Kliniken, mit überwiegenden Infektionen der oberen Atemwege. $45 \%$ waren Säuglinge, $32 \%$ hatten eine
Vorerkrankung. Bislang benötigten 3 Kinder (9\%) eine intensivstationäre Behandlung. Diskussion. COVID-19 bei hospitalisierten Kindern und Jugendlichen verläuft überwiegend als unkomplizierte febrile Erkrankung der oberen oder der unteren Atemwege. Nur sehr selten kommt es bei Kindern zu schweren Komplikationen oder Todesfällen. Weitere Informationen zum Verlauf von COVID-19 bei Neugeborenen, Säuglingen und Patienten mit Vorerkrankungen sowie zu therapeutischen und präventiven Maßnahmen werden dringend benötigt.

Schlüsselwörter

Coronavirus - SARS-CoV-2 - Akute respiratorische Infektion · Pädiatrie · Pneumonie

\section{COVID-19 in hospitalized children and adolescents. A systematic review on published case series (as of 31.03.2020) and first data from Germany}

\section{Abstract}

Background. The clinical knowledge about the course, complications and treatment of COVID-19 in children and adolescents is so far limited.

Aim. This systematic review summarizes the current scientific evidence regarding the clinical presentation of COVID-19 in hospitalized children based on available case series from China. In addition, first data from a nationwide pediatric hospital survey conducted by the German Society for Pediatric Infectious Diseases (DGPI) are presented. Methods. This study evaluated 12 case series from China with 6-2143 children infected with SARS-CoV-2, which were identified by a literature search in PubMed up to 31 March
2020. The database of the German nationwide DGPI COVID-19 survey was accessed on 6 April 2020.

Results. The median patient age in the case series was between 2 and 7 years and $18-45 \%$ were infants $<1$ year of age. The duration of hospital stay was 5-20 days. Most commonly reported symptoms were fever and cough; in $40-100 \%$ of cases involvement of the lower respiratory tract was reported, usually confirmed by computed tomography (CT). Severe and critical courses of disease were reported in up to $8 \%$ of the children including 2 fatalities. So far the German DGPI COVID19 survey reported 33 hospitalized children up to 6 April 2020, mostly with upper airway infections. Of these children, $45 \%$ were infants and $32 \%$ had an underlying medical condition. So far 3 children (9\%) needed admission to an intensive care unit.

Conclusion. COVID-19 in hospitalized children usually presented as an uncomplicated febrile upper airway infection or mild pneumonia. Severe cases or fatalities rarely occurred in children. Information on neonates and children with underlying chronic conditions as well as on therapeutic and preventive measures are urgently needed.

\section{Keywords}

Coronavirus · SARS-CoV-2 - Acute respiratory tract infection Pediatric $\cdot$ Pneumonia englischsprachigen Zusammenfassung berücksichtigt. Ein „Pre-print“-Artikel [23] ohne Peer Review wurde mitaufgenommen. Fallserien mit mehr als 5 hospitalisierten COVID-19-Patienten mit symptomatischer Infektion wurden eingeschlossen.

Demografische und klinische Daten wurden unabhängig von zwei der Verfasser extrahiert. Die Daten von 12 Fallserien wurden in Übersichtstabellen einzeln und für die 8 größten Studien zusam- mengefasst dargestellt. Falls der Anteil von Kindern mit Pneumonie von den Autoren nicht explizit ausgewiesen war, wurde der Nachweis von Veränderungen (milchglasartige bzw. fleckige Verschattungen sowie sonstige Auffälligkeiten) der Lunge in der Computertomographie (CT) sowie von Infiltraten in der Röntgenaufnahme als Kennzeichen einer Pneumonie gewertet. Die Einteilung des Schweregrades (asymptomatisch/ mild/moderat/schwer/kritisch) beruhte zumeist auf den Angaben/Definitionen der jeweiligen Autoren; für Studien mit entsprechend verfügbaren Details wurde anhand der klinischen Angaben analog der Definition von Dong et al. [10] nachträglich eine Zuordnung vorgenommen (Kriterien: s. Legende zu • Tab. 2). Die Normwertbereiche der jeweiligen lokalen Labore divergierten, daher wurde der jeweilige Anteil der Patienten mit abweichenden Laborwerten angegeben. 
Tab. 2 COVID-19 bei hospitalisierten Kindern und Jugendlichen (Daten aus publizierten Fallserien) - Klinische Charakteristika

Zusammen- Klinische Charakteristika

fassung ${ }^{\mathrm{a}}$

\begin{tabular}{|c|c|c|c|c|c|c|c|c|c|c|c|c|c|}
\hline $\begin{array}{l}\text { Autor } \\
\text { (Studiennr.) }\end{array}$ & $\begin{array}{l}\text { Studiennr. } \\
\text { (1)-(8) }\end{array}$ & $\begin{array}{l}\text { Dong } Y \\
\text { (1) }\end{array}$ & $\begin{array}{l}\operatorname{LuX} X \\
(2)\end{array}$ & $\begin{array}{l}\text { Ma H } \\
(3)\end{array}$ & $\begin{array}{l}\text { Qiu H } \\
\text { (4) }\end{array}$ & $\begin{array}{l}\text { Wang D } \\
\text { (5) }\end{array}$ & $\begin{array}{l}\text { Zheng } F \\
\text { (6) }\end{array}$ & $\begin{array}{l}\text { Xia W } \\
(7)\end{array}$ & $\begin{array}{l}\text { Cai J } \\
\text { (8) }\end{array}$ & $\begin{array}{l}\text { Zhou Y } \\
\text { (9) }\end{array}$ & $\begin{array}{l}\text { Wei M } \\
(10)\end{array}$ & $\begin{array}{l}\text { Sun D } \\
(11)\end{array}$ & $\begin{array}{l}\text { Liu W } \\
\text { (12) }\end{array}$ \\
\hline Patienten $(n)$ & $10-2143$ & $2143^{b}$ & 171 & 50 & 36 & 31 & 25 & 20 & 10 & $9^{c}$ & $9^{d}$ & $8^{e}$ & 6 \\
\hline \multicolumn{14}{|l|}{ Symptome } \\
\hline Fieber & $36-80 \%$ & $\mathrm{kA}$ & $42 \%$ & $64 \%$ & $36 \%$ & $65 \%$ & $52 \%$ & $60 \%$ & $80 \%$ & $44 \%$ & $44 \%$ & $75 \%$ & $100 \%$ \\
\hline Husten & $19-65 \%$ & $\mathrm{kA}$ & $49 \%$ & $44 \%$ & $19 \%$ & $45 \%$ & $44 \%$ & $65 \%$ & $60 \%$ & $22 \%$ & $22 \%$ & $75 \%$ & $100 \%$ \\
\hline Rhinitis & $8-40 \%$ & $\mathrm{kA}$ & $8 \%$ & $16 \%$ & $\mathrm{kA}$ & $\mathrm{kA}$ & $8 \%$ & $15 \%$ & $40 \%$ & $11 \%$ & $11 \%$ & $\mathrm{kA}$ & $17 \%$ \\
\hline Pharyngitis & $2-46 \%$ & $\mathrm{kA}$ & $46 \%$ & $2 \%$ & $6 \%$ & $\mathrm{kA}$ & $\mathrm{kA}$ & $5 \%$ & $40 \%$ & $\mathrm{kA}$ & $\mathrm{kA}$ & $13 \%$ & $83 \%$ \\
\hline Diarrhö & $0-15 \%$ & $\mathrm{kA}$ & $9 \%$ & $6 \%$ & $3 \%$ & $9 \%$ & $12 \%$ & $15 \%$ & $0 \%$ & $0 \%$ & $\mathrm{kA}$ & $38 \%$ & $0 \%$ \\
\hline Erbrechen & $4-10 \%$ & $\mathrm{kA}$ & $6 \%$ & $4 \%$ & $3 \%$ & $\mathrm{kA}$ & $8 \%$ & $10 \%$ & $\mathrm{kA}$ & $0 \%$ & $\mathrm{kA}$ & $50 \%$ & $67 \%$ \\
\hline Tachy-/Dyspnoe & $0-29 \%$ & $\mathrm{kA}$ & $29 \%$ & $\mathrm{kA}$ & $3 \%$ & $\mathrm{kA}$ & $8 \%$ & $10 \%$ & $0 \%$ & $\mathrm{kA}$ & $\mathrm{kA}$ & $100 \%$ & $17 \%$ \\
\hline Abgeschlagenheit & $4-10 \%$ & $\mathrm{kA}$ & $8 \%$ & $4 \%$ & $\mathrm{kA}$ & $10 \%$ & $\mathrm{kA}$ & $5 \%$ & $\mathrm{kA}$ & $0 \%$ & $\mathrm{kA}$ & $13 \%$ & $0 \%$ \\
\hline Pneumonie & $40-100 \%$ & $45 \%$ & $65 \%$ & $86 \%^{f}$ & $53 \%$ & $45 \%^{f}$ & $68 \%$ & $100 \%^{f}$ & $40 \%^{f}$ & $\geq 89 \%$ & $\mathrm{kA}$ & $100 \%$ & $67 \%$ \\
\hline \multicolumn{14}{|l|}{ Schweregrad $^{g}$} \\
\hline Asymptomatisch & $0-28 \%$ & $4,4 \%^{h}$ & $16 \%$ & $4 \%$ & $28 \%$ & $13 \%$ & $0 \%$ & $\mathrm{kA}$ & $0 \%$ & $\mathrm{kA}$ & $11 \%$ & - & $0 \%$ \\
\hline Mild & $10-60 \%$ & $51 \%$ & $19 \%$ & $10 \%$ & $19 \%$ & $42 \%$ & $32 \%$ & $\mathrm{kA}$ & $60 \%$ & $\mathrm{kA}$ & $22 \%$ & - & $33 \%$ \\
\hline Moderat & $39-82 \%$ & $39 \%$ & $62 \%$ & $82 \%$ & $53 \%$ & $45 \%$ & $60 \%$ & $\mathrm{kA}$ & $40 \%$ & $\mathrm{kA}$ & $\mathrm{kA}$ & - & $50 \%$ \\
\hline Schwer & $0-5 \%$ & $5 \%$ & $1 \%^{i}$ & $0 \%$ & $0 \%$ & $0 \%$ & $0 \%$ & $\mathrm{kA}$ & $0 \%$ & $0 \%$ & $0 \%$ & $63 \%$ & $17 \%$ \\
\hline Kritisch & $0-8 \%$ & $0,6 \%$ & $0,6 \%$ & $4 \%$ & $0 \%$ & $0 \%$ & $8 \%$ & $\mathrm{kA}$ & $0 \%$ & $0 \%$ & $0 \%$ & $38 \%$ & $0 \%$ \\
\hline $\begin{array}{l}\text { Todesfälle (Alter/ } \\
\text { Geschl.) }\end{array}$ & 2 & $\begin{array}{l}1 \text { (14 J./ } \\
\text { männl.) }\end{array}$ & $\begin{array}{l}1 \\
(10 \mathrm{M} . / \\
\text { weibl. })^{\mathrm{k}}\end{array}$ & 0 & 0 & 0 & 0 & 0 & 0 & 0 & 0 & 0 & 0 \\
\hline $\begin{array}{l}\text { Hospitalisie- } \\
\text { rungs- bzw. } \\
\text { Krankheits- } \\
\text { dauer (Tage) }\end{array}$ & $5-23$ & $\mathrm{kA}$ & $\mathrm{kA}$ & 5-19 & $\begin{array}{l}10-20 \\
7-22^{1}\end{array}$ & $7-23^{1}$ & $\mathrm{kA}$ & $8-20$ & $6-22^{1}$ & - & $\mathrm{kA}$ & $>9->28^{\mathrm{m}}$ & $5-13$ \\
\hline \multicolumn{14}{|c|}{ 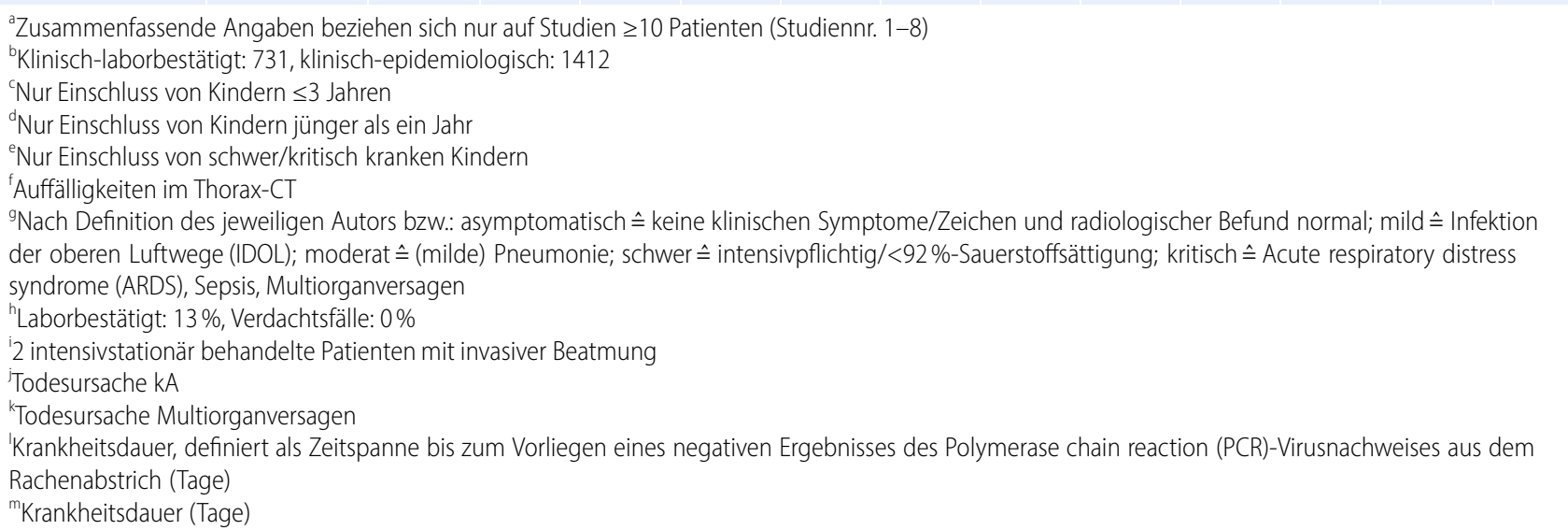 } \\
\hline
\end{tabular}

Zusätzlich wurden erste Daten zu stationär aufgenommenen Kindern aus Deutschland aus einem Survey der Deutschen Gesellschaft für Pädiatrische Infektiologie (DGPI) verfügbar (Stand 06.04.2020; COVID-19 Survey, https:// dgpi.de) [9].

\section{Resultate}

\section{Fallserien aus China}

\section{Ergebnisse der Literatursuche}

Von 219 identifizierten Artikeln wurden nach Inspektion des Titels und des Abstract insgesamt 75 Artikel näher geprüft. Insgesamt wurden 12 Fallserien mit hospitalisierten Kindern und Jugendlichen
( $n=6-2143)$ mit COVID-19identifiziert, die alle aus China stammten.

Insgesamt 8 Studien enthielten Angaben zu 10 oder mehr hospitalisierten Kindern. Die Studie von Dong et al. [10] zu allen bei der nationalen chinesischen Meldebörde bis zum 08.02.2020 registrierten Kindern mit COVID-19 $(n=2143)$ enthielt sowohl hospitalisierte als auch ambulante betreute Kinder. 

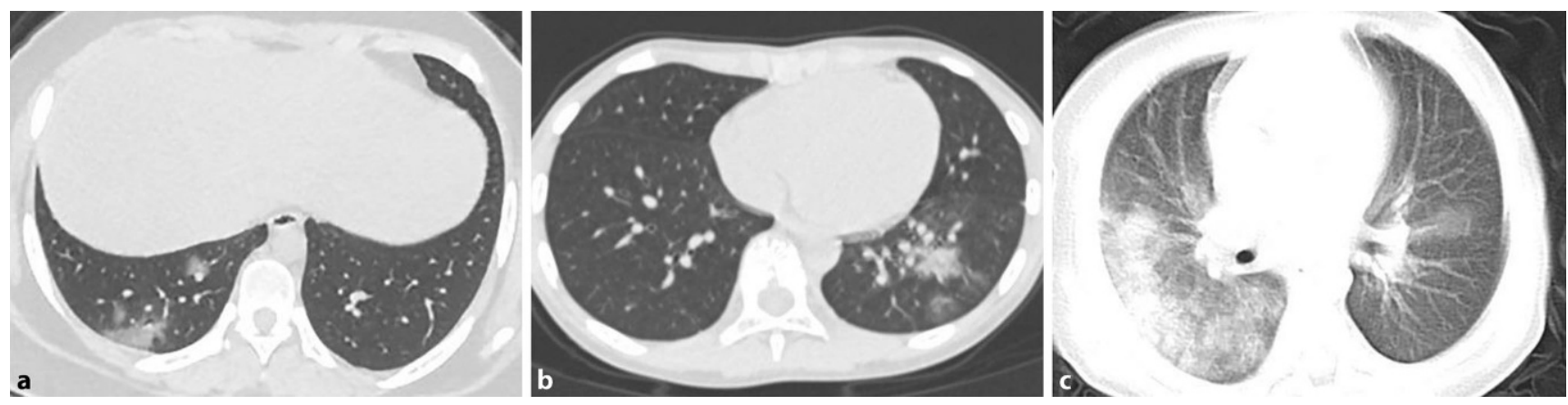

Abb. 1 A Typische Befunde des Thorax-CT für Kinder mit COVID-19-Pneumonie. a Weiblich, 14 Jahre alt: vereinzelte milchglasartige Verschattung im rechten unteren Lungenlappen, Lokalisation pleuranah und -fern. b Männlich, 10 Jahre alt: Konsolidierungen mit "halo sign“ im linken unteren Lungenlappen, umgeben von milchglasartigen Verschattungen. c Männlich, ein Jahr alt: diffuse Konsolidierungen und milchglasartige Verschattungen in beiden Lungen, zusätzlich „white lung" rechts. (Aus Xia et al. 2020 [32]; Abdruck mit freundlicher Genehmigung des Verlags John Wiley \& Sons)

Die Fallserie von Liu et al. [19] beschreibt die ersten 6 aus Wuhan dokumentierten Fälle von COVID-19 bei Kindern. Drei kleinere Fallserien (8 bis $9 \mathrm{~Pa}$ tienten) bezogen sich auf Säuglinge [30], Säuglinge und Kleinkinder [35] und intensivstationär behandelte Kinder [28].

Sechs Studien stammten aus dem Wuhan Children's Hospital [19, 21, 23, 28, 32] bzw. der entsprechenden Provinz Hubei [34], davon 5 aus annähernd demselben Zeitraum Mitte/Ende Januar bis ca. Mitte/Ende Februar [21, 23, 28, 32, 34]. Diese Studien zeigten teilweise Überschneidungen insbesondere in der Angabe von Kindern mit spezifischen Vorerkrankungen, Koinfektionen, schwer und kritisch kranken Kindern bzw. Todesfällen.

\section{Demografische Daten, Inkubati- ons- und Hospitalisierungsdauer, Vorerkrankungen}

Insgesamt umfassten die 12 Studien 2518 Patienten aus dem gesamten pädiatrischen Altersspektrum. Bei den 8 Fallserien mit mindestens 10 hospitalisierten Kindern lag der Altersmedian im Bereich von 2 bis 7 Jahren. Der Anteil von Säuglingen unter einem Jahr war 18-45\%. Der Anteil der männlichen Kinder lag insgesamt bei $40-65 \%$, bei 27 Säuglingen (zusammengefasst aus 4 Studien) bei $41 \%[2,23,28,30]$.

In 2 Studien wird die Inkubationszeit bei Kindern mit 2 bis 10 Tagen angegeben, mit einem Median von 6 bis 7 Tagen [2, 28]. Die Hospitalisierungsdauer lag bei
5 bis 20 Tagen, bei intensivstationären Patienten bei bis zu über 28 Tagen [28]. Vorerkrankungen wurden bei 12 (12\%) von 98 Patienten in 6 Studien berichtet: 8 Kinder mit kardiologischer (davon 6 in der Studie von Xia et al. [32]), 2 Kinder mit hämatologisch-onkologischer [21, 28] und je ein Kind mit nephrologischer und neurologischer Vorerkrankung.

Übersicht: - Tab. 1.

\section{Klinische Charakteristika Übersicht: • Tab. 2.}

Symptome. Das häufigste Symptom war Fieber (36-80\%), mit einer mittleren Dauer von 2 bis 3 Tagen [2, 21, 26] und einem Bereich von einem bis 16 Tagen [21, 29]. Der Anteil der Kinder mit Fieber $\geq 38,5^{\circ} \mathrm{C}$ lag bei $11-49 \%[2,26]$, mit hohem Fieber $>39^{\circ} \mathrm{C}$ bei $9 \%$ [21]. Häufig waren auch (trockener) Husten (19-65\%), Rhinitis (8-40\%) und Pharyngitis $(2-46 \%)$. Eine Studie erfasste eine Tachykardie bei $42 \%$ von 171 Kindern [21]. Bis zu 29\% zeigten eine Tachypnoe/Dyspnoe, 0-15\% gastrointestinale Symptome (Erbrechen, Diarrhö, Bauchschmerzen), 4-10\% Abgeschlagenheit und 4-8\% Kopfschmerzen [23, 26]. Bei Säuglingen und Kleinkindern wurden ebenfalls Fieber, Husten und Rhinitis als häufigste Symptome genannt [2, 23, 30, 35].

Pneumonie. Eine Pneumonie bzw. ein Nachweis von Auffälligkeiten bzw. Verschattungen der Lunge im CT lag bei
$40-100 \%$ der Kinder vor. Davon zeigten 11-42\% kein Fieber oder Husten bzw. sonstige Symptome einer Infektion [21, 26].

In der Studie von $\mathrm{Lu}$ et al. [21] mit 111 Kindern mit Angabe einer Pneumonie waren in jeder pädiatrischen Altersgruppe $(<1,1-5,6-10$ und 11-15 J) zwischen 23 und $31 \%$ betroffen. In der Studie von Zheng et al. [34] war der Anteil von Kindern mit abnormalem CT am höchsten bei Kindern unter 3 Jahren ( 8 von 10 Kindern <3 J), inklusive zweier kritisch erkrankter Kinder.

Eine Sauerstofftherapie war nur selten notwendig (0-17\%); eine invasive Beatmung auf Intensivstation erfolgte bei 0-8\% der Patienten.

Schweregrad. Der Anteil asymptomatischer Patienten lag in den 5 größten Studien bei 4-28\%. Die Krankheit verlief bei 10-60\% der Kinder mild, vorwiegend als fieberhafte Erkrankung der oberen Atemwege. Mit 39-82\% am häufigsten war ein moderater Krankheitsverlauf mit leichter Pneumonie. Einen schweren oder sehr schweren Verlauf zeigten bis zu $5 \%$ bzw. $8 \%$ der hospitalisierten Kinder.

In der großen Studie von Dong et al. [10] mit 2143 Patienten waren $60 \%$ der schwer bzw. $69 \%$ der kritisch Erkrankten in der Altersgruppe unter 5 Jahren. $11 \%$ der Säuglinge bzw. 7 \% der Ein- bis 5-Jährigen mit COVID-19 zeigten hier einen schweren bis sehr schweren Verlauf, im Vergleich zu 3-4\% bei älteren Kindern und Jugendlichen. 


\section{Übersichten}

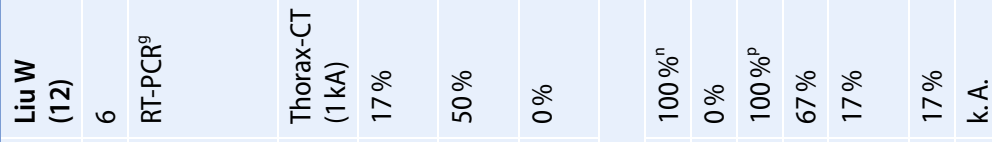

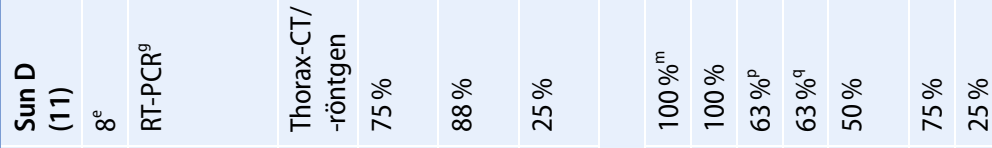

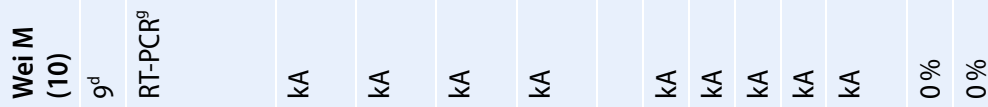

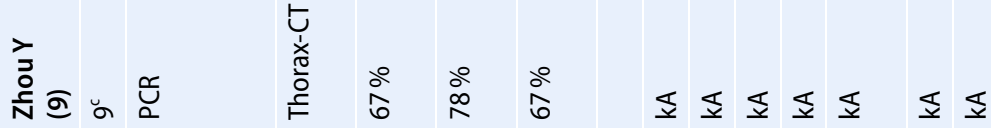

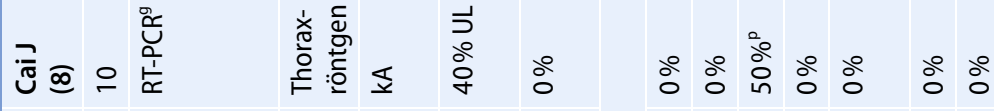

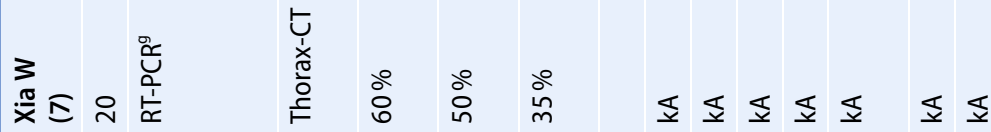

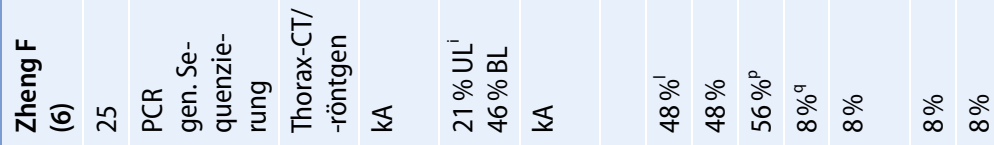

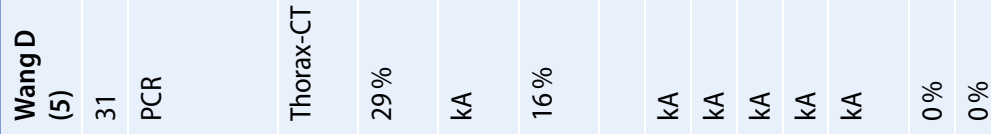

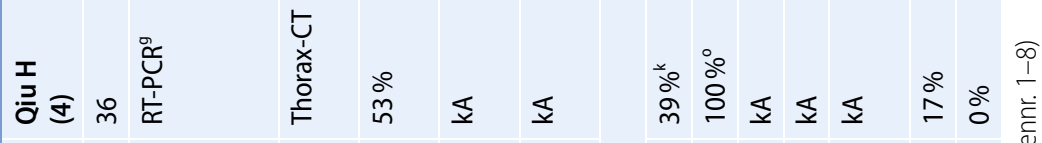

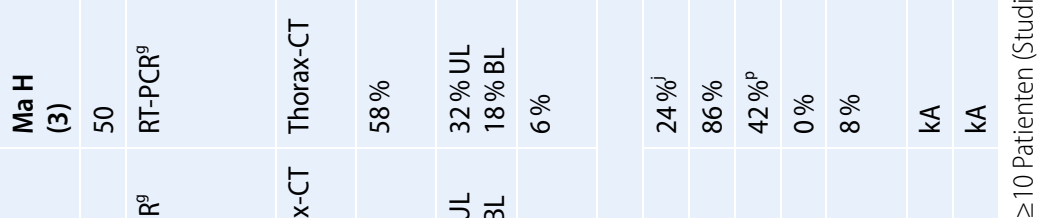

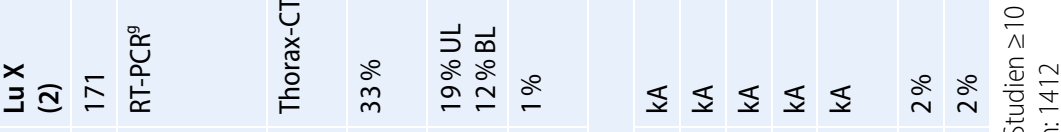
خे

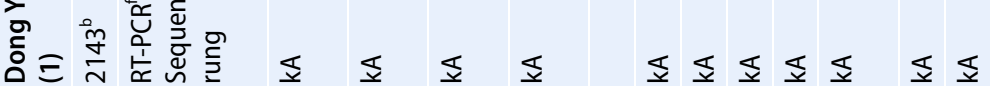

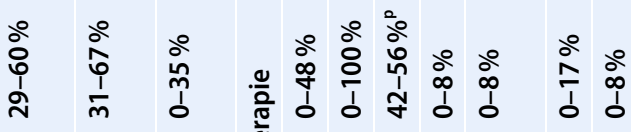


Tab. 4 COVID-19 bei hospitalisierten Kindern und Jugendlichen (Daten aus publizierten Fallserien) - Labor

\begin{tabular}{|c|c|c|c|c|c|c|c|c|c|c|c|c|c|}
\hline \multirow[b]{2}{*}{$\begin{array}{l}\text { Autor } \\
\text { (Studiennr.) }\end{array}$} & \multirow{2}{*}{$\begin{array}{l}\begin{array}{l}\text { Zusammen- } \\
\text { fassung }^{a}\end{array} \\
\begin{array}{l}\text { Studiennr. } \\
(1)-(8)\end{array}\end{array}$} & \multicolumn{12}{|l|}{ Labor $^{b}$} \\
\hline & & $\begin{array}{l}\text { Dong } Y \\
\text { (1) }\end{array}$ & $\begin{array}{l}\operatorname{Lu} X \\
\text { (2) }\end{array}$ & $\begin{array}{l}\mathrm{MaH} \\
(3)\end{array}$ & $\begin{array}{l}\text { Qiu H } \\
\text { (4) }\end{array}$ & $\begin{array}{l}\text { Wang D } \\
\text { (5) }\end{array}$ & $\begin{array}{l}\text { Zheng } F \\
\text { (6) }\end{array}$ & $\begin{array}{l}\text { Xia W } \\
\text { (7) }\end{array}$ & $\begin{array}{l}\text { Cai J } \\
\text { (8) }\end{array}$ & $\begin{array}{l}\text { Zhou Y } \\
\text { (9) }\end{array}$ & $\begin{array}{l}\text { Wei M } \\
(10)\end{array}$ & $\begin{array}{l}\text { Sun D } \\
\text { (11) }\end{array}$ & $\begin{array}{l}\text { Liu W } \\
\text { (12) }\end{array}$ \\
\hline Patienten $(n)$ & $10-2143$ & $2143^{c}$ & 171 & 50 & 36 & 31 & 25 & 20 & 10 & $9^{d}$ & $9^{e}$ & $8^{f}$ & 6 \\
\hline \multicolumn{14}{|l|}{ Leukozyten } \\
\hline Leukozytose & $4-30 \%$ & $\mathrm{kA}$ & $\mathrm{kA}$ & $4 \%$ & $\mathrm{kA}$ & $10 \%^{9}$ & $\begin{array}{l}(50 \% \\
[1 / 2])^{h}\end{array}$ & $10 \%$ & $30 \%$ & $22 \%$ & $\mathrm{kA}$ & $25 \%$ & $0 \%$ \\
\hline Leukopenie & $6-38 \%$ & $\mathrm{kA}$ & $26 \%$ & $38 \%$ & $19 \%$ & $6 \%$ & $\begin{array}{l}(0 \% \\
[0 / 2])^{\mathrm{h}}\end{array}$ & $20 \%$ & $10 \%$ & $0 \%$ & $\mathrm{kA}$ & $13 \%$ & $67 \%$ \\
\hline \multicolumn{14}{|l|}{ Lymphozyten } \\
\hline Lymphozytose & $8-20 \%$ & $\mathrm{kA}$ & $\mathrm{kA}$ & $8 \%$ & $\mathrm{kA}$ & $13 \%^{9}$ & $\mathrm{kA}$ & $15 \%$ & $20 \%$ & $67 \%$ & $\mathrm{kA}$ & $25 \%$ & $0 \%$ \\
\hline Lymphopenie & $0-40 \%$ & $\mathrm{kA}$ & $4 \%$ & $20 \%$ & $31 \%$ & $6 \%$ & $40 \%$ & $35 \%$ & $0 \%$ & $0 \%$ & $\mathrm{kA}$ & $13 \%$ & $100 \%$ \\
\hline \multicolumn{14}{|l|}{ Thrombozyten } \\
\hline Thrombozytose & $6-20 \%$ & $\mathrm{kA}$ & $\mathrm{kA}$ & $16 \%$ & $\mathrm{kA}$ & $6 \%$ & $\mathrm{kA}$ & $\mathrm{kA}$ & $20 \%$ & $\mathrm{kA}$ & $\mathrm{kA}$ & $25 \%$ & $0 \%$ \\
\hline Thrombopenie & $10-16 \%$ & $\mathrm{kA}$ & $\mathrm{kA}$ & $16 \%$ & $\mathrm{kA}$ & $\mathrm{kA}$ & kA & $\mathrm{kA}$ & $10 \%$ & $\mathrm{kA}$ & $\mathrm{kA}$ & $13 \%$ & $0 \%$ \\
\hline CRP erhöht & $3-35 \%$ & $\mathrm{kA}$ & $20 \%$ & $18 \%$ & $3 \%$ & $\begin{array}{l}10 \% \\
(3 / 30)\end{array}$ & $\begin{array}{l}(50 \% \\
[1 / 2])^{h}\end{array}$ & $35 \%^{i}$ & $30 \%$ & $\mathrm{kA}$ & $\mathrm{kA}$ & $63 \%$ & $83 \%$ \\
\hline PCT erhöht & $4-80 \%$ & $\mathrm{kA}$ & $64 \%$ & $\mathrm{kA}$ & $17 \%$ & $\begin{array}{l}4 \% \\
(1 / 28)\end{array}$ & $\mathrm{kA}$ & $80 \%$ & $10 \%$ & $\mathrm{kA}$ & $\mathrm{kA}$ & $63 \%$ & $\mathrm{kA}$ \\
\hline $\begin{array}{l}\text { BSG be- } \\
\text { schleunigt }\end{array}$ & $0-19 \%$ & $\mathrm{kA}$ & $\mathrm{kA}$ & $\mathrm{kA}$ & $0 \%$ & $\begin{array}{l}19 \% \\
(4 / 21)\end{array}$ & $\mathrm{kA}$ & $\mathrm{kA}$ & $\mathrm{kA}$ & kA & $\mathrm{kA}$ & $\mathrm{kA}$ & $\begin{array}{l}40 \% \\
(2 / 5)\end{array}$ \\
\hline ALT erhöht & $6-25 \%$ & $\mathrm{kA}$ & $12 \%$ & $\mathrm{kA}$ & $6 \%$ & $\begin{array}{l}22 \% \\
(6 / 27)\end{array}$ & $\mathrm{kA}$ & $25 \%$ & $10 \%$ & $\mathrm{kA}$ & $\mathrm{kA}$ & $50 \%$ & $17 \%$ \\
\hline AST erhöht & $8-22 \%$ & $\mathrm{kA}$ & $15 \%$ & $\mathrm{kA}$ & $8 \%$ & $\begin{array}{l}22 \% \\
(6 / 27)\end{array}$ & $\mathrm{kA}$ & $\mathrm{kA}$ & $20 \%$ & $\mathrm{kA}$ & $\mathrm{kA}$ & $0 \%$ & $67 \%$ \\
\hline CK erhöht & $3-15 \%$ & $\mathrm{kA}$ & $\mathrm{kA}$ & $\mathrm{kA}$ & $3 \%$ & $\begin{array}{l}15 \% \\
(4 / 27)\end{array}$ & $\begin{array}{l}(100 \% \\
[2 / 2])^{\mathrm{h}}\end{array}$ & $\mathrm{kA}$ & $\mathrm{kA}$ & $\mathrm{kA}$ & $\mathrm{kA}$ & $25 \%$ & $0 \%$ \\
\hline CK-MB erhöht & $25-50 \%$ & $\mathrm{kA}$ & $\mathrm{kA}$ & $\mathrm{kA}$ & $31 \%$ & $\mathrm{kA}$ & $\mathrm{kA}$ & $25 \%{ }^{i}$ & $50 \%$ & $\mathrm{kA}$ & $\mathrm{kA}$ & $\mathrm{kA}$ & $\mathrm{kA}$ \\
\hline LDH erhöht & $30 \%$ & $\mathrm{kA}$ & $\mathrm{kA}$ & $\mathrm{kA}$ & $\mathrm{kA}$ & $\mathrm{kA}$ & $\begin{array}{l}(100 \% \\
[2 / 2])^{\mathrm{h}}\end{array}$ & $\mathrm{kA}$ & $30 \%$ & $\mathrm{kA}$ & $\mathrm{kA}$ & $63 \%$ & $33 \%$ \\
\hline $\begin{array}{l}\text { D-Dimere } \\
\text { erhöht }\end{array}$ & $8-40 \%$ & $\mathrm{kA}$ & $14 \%$ & $\mathrm{kA}$ & $8 \%$ & $\mathrm{kA}$ & $\mathrm{kA}$ & $\mathrm{kA}$ & $\begin{array}{l}40 \% \\
(2 / 5)\end{array}$ & $\mathrm{kA}$ & $\mathrm{kA}$ & $\begin{array}{l}20 \% \\
(2 / 5)\end{array}$ & $50 \%$ \\
\hline \multicolumn{14}{|c|}{ 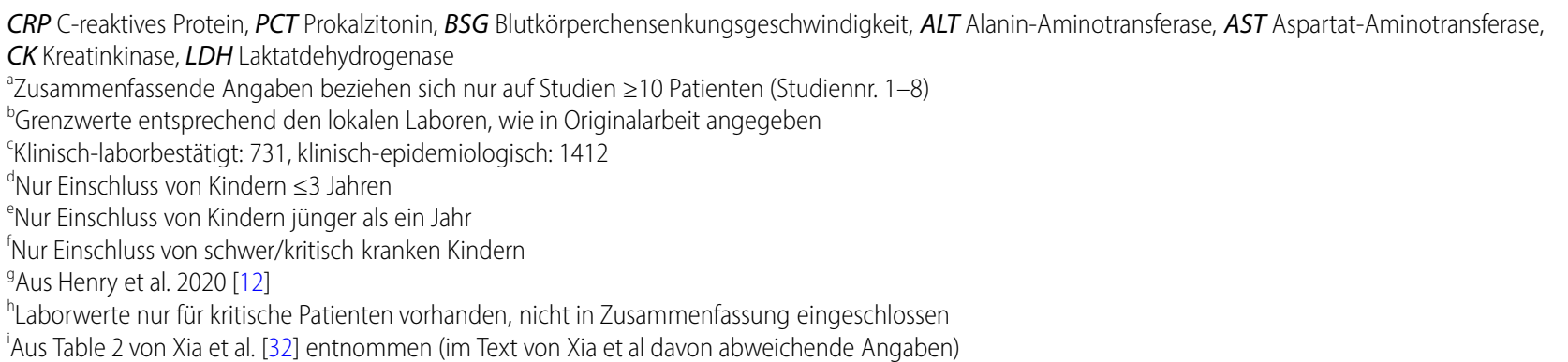 } \\
\hline
\end{tabular}

Intensivmedizinisch behandelte Patienten und Todesfälle. Insgesamt liegen zu 11 intensivmedizinisch behandelten Kindern (Altersmedian 2 Jahre, Bereich 2 Monate bis 15 Jahre; 8 von 11 männlich) detaillierte Beschreibungen vor [19, 28, 34]. Darunter waren 3 Säuglinge jünger als ein Jahr, 4 Kleinkinder $\leq 3$ Jahren und 4 Schulkinder. Bei 4 Kindern war eine Grunderkrankung bekannt, hierunter 2 Säuglinge mit angeborenen Herzfehlern, ein 8-jähriger Junge mit akuter lymphatischer Leukämie und ein einjähriger Junge mit Hydronephrose [28, 34].

Die beiden kritischen Patienten mit angeborenen Herzfehlern zeigten Fieber, Dyspnoe und Husten (männl., 8Mo.) bzw. Diarrhö (männl., ein J.) sowie radiologisch bilaterale Konsolidierungen in der Lunge. Im Verlauf entwickelten beide ein ARDS und mussten invasiv beatmet werden. Als zusätzliche Komplikationen wurden bei dem Einjährigen eine Hyponatriämie (Natrium $126 \mathrm{mmol} / \mathrm{l}$ ) sowie ein dialysepflichtiges akutes Nierenversagen beschrieben [34].

Ein 3-jähriges Mädchen mit schwerem Verlauf litt unter hohem Fieber $\left(>39^{\circ} \mathrm{C}\right)$ über 11 Tage, Husten, Tonsillitis, Erbrechen und Tachypnoe. Im CT-Bild des Thorax zeigten sich beidseits fleckige, milchglasartige Verschattungen [19]. 
Von den 8 intensivpflichtigen Kindern der Fallserie von Sun et al. [28] zeigten alle pulmonale Komplikationen mit einoder meist beidseitigen (6 von 8 Patienten) Auffälligkeiten in der Bildgebung (milchglasartige Verschattungen 6 von 8 , fleckige Verschattungen 6 von 8 , „white lung“ im Verlauf 1 von 8). Insgesamt 6 der 8 Kinder erhielten eine Sauerstofftherapie, 2 Kinder wurden zusätzlich invasiv beatmet. Zwei Patienten entwickelten ein septisches Geschehen bis zum septischen Schock mit Multiorganversagen, hierunter ein 13 Monate altes männliches Kleinkind mit Herzinsuffizienz, Koagulopathie, Nierensteinen und Hydronephrose [28], sowie ein im Verlauf verstorbenes 10 Monate altes Mädchen mit einer toxischen Enzephalopathie, Status epilepticus, disseminierter intravasaler Gerinnung und Invagination [21, 23, 28]. In der Arbeit von Dong et al. [10] wurde ein weiterer Todesfall eines 14-jährigen Jungen ohne weitere Informationen berichtet.

\section{Diagnostik}

Übersicht: • Tab. 3.

Erregernachweise. SARS-CoV-2 wurde zumeist mittels Reverse-Transkriptase(RT)-PCR detektiert. Koinfektionen mit Influenzavirus (6), RSV (2), Zytomegalievirus (1), Mykoplasmen (12) sowie Enterobacter aerogenes wurden bei $12-40 \%$ der Patienten aus 4 Studien beschrieben [23, 28, 32, 34].

SARS-CoV-2 konnte in respiratorischen Kontrollabstrichen nach 6 bis 22 Tagen nicht mehr nachgewiesen werden. In einer Studie wurden auch Stuhlproben von 6 Kindern untersucht, bei einem der älteren Kinder (10 J.) war die Testung nach 10 Tagen negativ, aber bei 5 Kindern "viral shedding“ für mindestens 18 bis 30 Tage nachweisbar; Serum und Urinproben dieser 5 Patienten waren negativ [2].

Radiologie. Die meisten Informationen liegen zu CT-Aufnahmen des Thorax vor. Typisches Zeichen der SARS-CoV2-positiven Kinder in der CT-Diagnostik waren milchglasartige (29-60\%) und fleckige Verschattungen (gesamt 31-67\%; 19-40\% unilateral, $12-46 \%$ bilateral). Bei bis zu $35 \%$ der Kinder konnten weitere Auffälligkeiten in der CT-Bildgebung beschrieben werden: Konsolidierungen mit "halo sign" (10 von 20 Patienten, bzw. 3 von 9), kleine Knötchen (3 von 20, bzw. 6 von 9), retikuläre Zeichnungsvermehrung (4 von 20) und Pleuraerguss (1 von 50) [21, 23, 32, 35]. Typische Aufnahmen zeigt - Abb. 1 (aus Xia et al. [32]).

Nach Ma et al. [23] finden sich die COVID-19-assoziierten Verschattungen meist pleuranah (82\%) und in den Unterlappen (56\%). 12 von 15 Kindern zeigten bei Entlassung (im Durchschnitt nach 8 Tagen) noch radiologische Auffälligkeiten im Kontroll-CT des Thorax, trotz fehlender klinischer Symptome [23]. Eine weitere Studie wies ebenfalls pulmonale Auffälligkeiten (milchglasartige Verschattungen) bei 8 asymptomatischen von insgesamt 19 CT-untersuchten Kindern nach [26]. In der Studie von Xia et al. [32] waren ebenfalls noch CTAuffälligkeiten nach Abklingen der klinischen Symptomatik und 2 negativen Virusnachweisen sichtbar.

\section{Labor. Übersicht: • Tab. 4.}

In den 8 größeren Studien waren die absoluten Zahlen der Leukozyten, Lymphozyten sowie Thrombozyten bei der Mehrheit der Kinder normwertig. Leukozytose und Leukopenie wurden unabhängig vom Schweregrad bei 4-30\% bzw. 6-38\% beobachtet, eine Lymphopenie bei $0-40 \%$ und eine Lymphozytose bei 8-20\%. Die Zahl der Thrombozyten war bei 6-20\% der Kinder erhöht und bei 10-16\% erniedrigt. Die Blutbildauffälligkeiten bei schwer und kritisch erkrankten Kindern waren vergleichbar [19, 28, 34].

Als Entzündungsparameter wurden C-reaktives Protein (CRP in 9 von 12 Publikationen), Prokalzitonin (PCT in 6 von 12) und Blutkörperchensenkungsgeschwindigkeit (BSG in 3 von 12) berichtet. Das CRP war bei 3-35\% meist leicht erhöht, die BSG war bei bis zu 19\% der Kinder leicht erhöht. Prokalzitonin war je nach Fallserie bei $4-80 \%$ der Kinder erhöht. CRP und PCT stiegen bei Kindern mit schweren und kritischen Verläufen z. T. deutlich an [19, 28, 34].

Eine leichtgradige Erhöhung der Transaminasenaktivität wurde bei 6-25\%
(Alanin-Aminotransferase, ALT) und 8-22\% (Aspartat-Aminotransferase, AST) der Kinder beobachtet. Leichtgradige Erhöhungen der Kreatinkinase (CK) wurden in 4 Arbeiten bei 3-15\% der Kinder berichtet, sowie bei $36 \%$ (4 von 11) aller Patienten auf Intensivstation. Ein im Verlauf verstorbener Säugling verzeichnete einen CK-Anstieg auf $20.702 \mathrm{U} / \mathrm{l}$. Eine erhöhte, für die Herzmuskulatur spezifischere CK-MB wurde in 3 Arbeiten bei $25-50 \%$ der Patienten angegeben. Ein Anstieg der Laktatdehydrogenase (LDH) (361-891 U/l) wurde bei $73 \%$ (8 von 11) der Kinder mit schwereren Verläufen beobachtet [19, 28, 34]. D-Dimer-Bestimmungen wurden in 5 Arbeiten angegeben und waren bei $8-40 \%$ der Patienten sowie bei $50 \%$ (3 von 6, [19, 28]) aller intensivpflichtigen Kinder erhöht. Neben überwiegend leichten Erhöhungen ist bei dem verstorbenen Säugling ein Anstieg der D-Dimere auf bis zu 40,34 mg/l dokumentiert [21].

In der Studie von Qiu et al. [26] zeigten Kinder mit Pneumonie gegenüber Kindern mit asymptomatischer/milder Erkrankung häufiger eine Lymphozytopenie sowie Erhöhungen für Prokalzitonin, D-Dimere und CK-MB.

\section{Medikamentöse Therapie}

Angaben zur symptomatischen, antiinflammatorischen und antiviralen Therapie sind nur teilweise vorhanden. In $3 \mathrm{der}$ größeren Studien [23, 26, 34] erhielten $48-100 \%$ der Patienten Interferon und 24-48\% antivirale Medikamente (Lopinavir/Ritonavir bzw. Litonavir, Ribavirin, Oseltamivir, Ganciclovir, Umifenovir). In 2 der Studien wurden Immunglobuline jeweils $8 \%$ der Patienten verabreicht, in einer Studie Glukokortikoide (8\%). Eine antibiotische Therapie wurde $42-56 \%$ der Kinder verabreicht. Die intensivmedizinisch behandelten Patienten bei Sun et al. [28] erhielten alle Oseltamivir, Ribavirin und Interferon, Antibiotika in $63 \%$, Glukokortikoide in $63 \%$ und Immunglobuline i.v. in $50 \%$. Bewertungen zur Wirksamkeit der Behandlungen liegen nicht vor. 


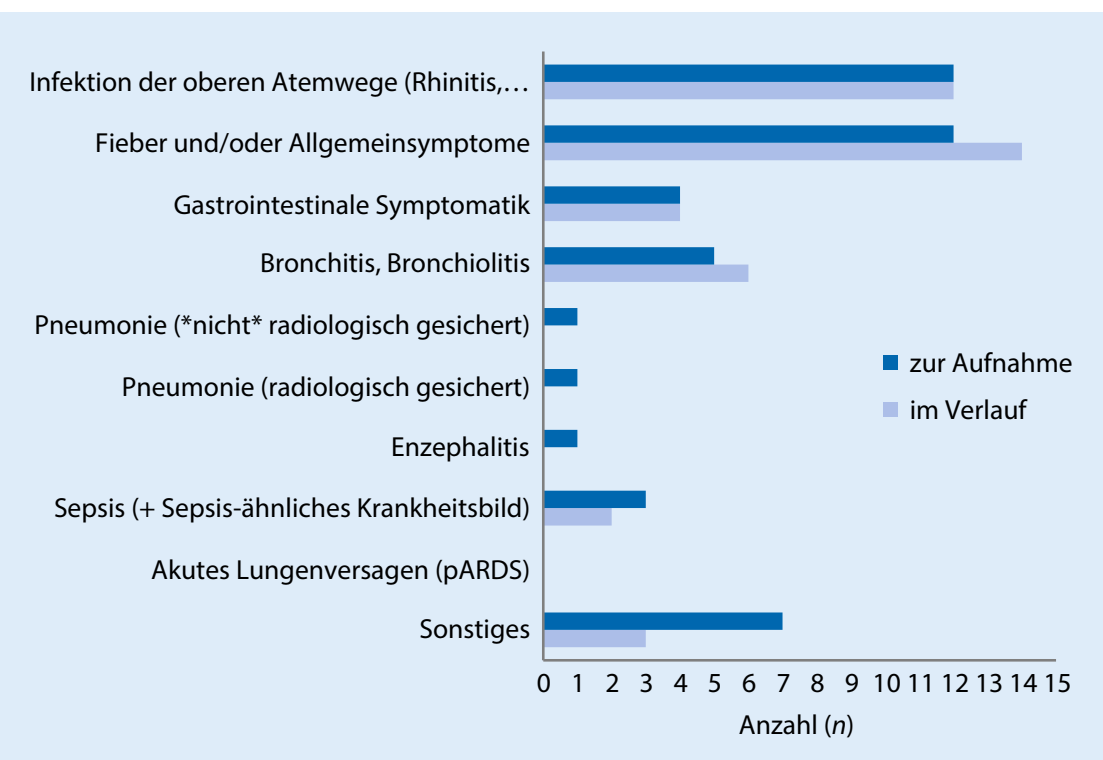

Abb. 2 \ COVID-19 bei 33 hospitalisierten Kindern in Deutschland: Symptome bei Aufnahme und im Verlauf. Mehrfachnennung möglich. (Aus DGPI [9], Stand 06.04.2020)

\section{Hospitalisierungen in Deutschland (DGPI-Survey)}

Seit dem 18.03.2020 werden im Survey der DGPI - in enger Kooperation mit DGKJ, VLKKD und vielen Konventgesellschaften - Fälle aus ganz Deutschland registriert. Im Zeitraum bis zum 06.04.2020 wurden dort Aufnahmen von 33 Kindern aus 21 Kliniken erfasst, davon 3 auf Intensivstation. Von den 33 Kindern waren 15 (45\%) Säuglinge (darunter 5 Neugeborene), 12 Kinder im Alter von einem bis 5 Jahren, ein Kind in der Altersgruppe von 6 bis 10 Jahren und 5 Kinder über 11 Jahren; 22 von 33 (67\%) waren weiblich. Die häufigsten Symptome waren Fieber und/oder Allgemeinsymptome (14 von 33; $42 \%$ ), Infektion der oberen Atemwege (12 von 33; 36\%) und gastrointestinale Symptome (4 von 33; $11 \%)$. Eine Bronchitis/Bronchiolitis wurde bei 6 Kindern (18\%), Pneumonie bei 2 Kindern (6\%), Sepsis bei $3(9 \%)$ und Enzephalitis bei einem Kind (3\%) berichtet (•Abb.2). Von 22 Kindern mit verfügbaren Angaben hatten 7 (32\%) Kinder kardiale (3), pulmonale (4), neurologische (3) oder andere (6) Vorerkrankungen. Therapeutisch wurden eine Sauerstoffsupplementation bei 3 von 33 Kindern $(9 \%)$ sowie eine nichtinvasive Beatmung bei einem Kind durchgeführt (3\%). Medikamentös wurden inhalative Bron-
Verlauf sowie die Diagnostik und Therapie von COVID-19 bei Kindern noch als begrenzt angesehen werden. Obwohl alle 12 Fallserien vorwiegend hospitalisierte Kinder eingeschlossen haben, sind sie in ihrem methodischen Aufbau, der Qualität der Datenerhebung, der Auswahl der Patientenuntergruppen und der Repräsentativität für die Pädiatrie sehr unterschiedlich. Daher müssen jeder Vergleich der Fallserien untereinander sowie jede Schlussfolgerung aus diesen Veröffentlichungen mit Vorsicht interpretiert werden.

\section{Klinische Symptomatik und Verlauf}

Nach derzeitigen Erkenntnissen infizieren sich Kinder und Jugendliche nicht seltener als Erwachsene mit SARS-CoV-2, ihre Infektion scheint aber oft asymptomatisch oder oligosymptomatisch abzulaufen [22]. Die hier vorgestellten Fallserien bestätigen die früheren Beobachtungen, dass auch symptomatische Infektionen bei hospitalisierten Kindern generell weniger schwer und mit einer besseren Prognose als bei Erwachsenen verlaufen $[11,22,26,31,36]$. Typische Kennzeichen einer COVID-19-Infektion bei hospitalisierten Kindern waren Fieber und eine Infektionserkrankung der oberen Atemwege mit Rhinitis, Pharyngitis und Husten oder der unteren Atemwege mit Pneumonie und/oder Bronchitis. In einer Gegenüberstellung in der Studie von $\mathrm{Ma}$ et al. [23] zeigten die erkrankten Kinder ein ähnliches Spektrum an Symptomen wie Erwachsene, aber der Anteil der Patienten mit Fieber (64\% vs. $88 \%$ ), Husten $(44 \%$ vs. $68 \%)$, Halsschmerzen ( $2 \%$ vs. $14 \%$ ) und Abgeschlagenheit (4\% vs. $38 \%$ ) war bei Kindern deutlich niedriger. Zusätzlich berichten Qiu et al. [26] von einem selteneren Auftreten von Pneumonie ( $53 \%$ vs. $95 \%)$, von seltener erhöhtem CRP (3\% vs. $49 \%$ ) und von selteneren schweren Verläufen ( $0 \%$ vs. $23 \%$ ). Intensivstationäre Behandlungen wurden bei Kindern selten berichtet [10, 28].

Bei Kindern $<6$ Jahre bestand jedoch ein etwas erhöhtes Risiko für einen schwereren Verlauf [10]. Die Wirksamkeit der häufig durchgeführten antiviralen und antiinflammatorischen Therapie ließ sich aufgrund der fehlenden Kon- 
trollen nicht beurteilen. In Deutschland kam es seit dem 23.03.2020 zu einer deutlichen Zunahme der dem DGPISurvey gemeldeten stationären Aufnahmen, mit einem Kind (KW 11) bzw. 14 (KW 12) SARS-CoV-2 infizierten Kindern pro Woche mit überwiegend leichtem klinischen Verlauf [9].

\section{Todesfälle im Kindes- und Jugendalter}

In den hier untersuchten chinesischen Fallserien wurden insgesamt nur 2 Todesfälle berichtet $[10,21]$. Weder im DGPI-Survey [9] noch durch das Robert Koch-Institut [27] wurde bis zum 06.04.2020 ein Todesfall bei Kindern gemeldet. Zum 03.04.2020 weisen die Meldestatistiken der italienischen Gesundheitsbehörde (Istituto Superiore di Sanità) [14] bei insgesamt 13.241 COVID-19-Verstorbenen einen Patienten im Alter unter 20 Jahren auf. In Spanien wurde am 05.04.2020 bei insgesamt 4709 Todesfällen von einem verstorbenen $\mathrm{Pa}$ tienten unter 10 Jahren sowie einem verstorbenen Patienten im Alter von 10 bis 19 Jahren berichtet [24]. In den USA wurde am 28.03.2020 der Tod eines Säuglings von der Gesundheitsbehörde von Illinois veröffentlicht [13]. Noch unbestätigte Pressemeldungen betrafen die Tode einer 16-Jährigen in Frankreich, eines 14-Jährigen in Portugal, eines 13Jährigen aus Großbritannien und einer 12-Jährigen aus Belgien.

\section{Neugeborene und Säuglinge}

$\mathrm{Zu}$ COVID-19-Erkrankungen bei Neugeborenen gibt es bislang nur wenige Einzelfallberichte $[15,33]$. In der Studie von Xia et al. [32] wurden 3 Neugeborene, in einem Review aus China 2 Neugeborenen mit COVID-19-Erkrankungen genannt [20], darunter ein 17 Tage alter Junge mit Fieber, Husten und Erbrechen (Infektion im Haushalt) und ein 5 Tage altes Kind mit Fieber (Mutter infiziert). Von Neugeborenen, die von COVID19-erkrankten Müttern geboren wurden, ist bisher nur über ein infiziertes, aber asymptomatisches Kind berichtet worden [20]. Bislang gibt es keine Belege für eine vertikale Transmission von CO-
VID-19 [16, 17, 25]. Interessanterweise waren im DGPI-Survey unter den ersten 26 stationären Aufnahme insgesamt 15 Säuglinge (45\%), darunter $5 \mathrm{im} \mathrm{Neu-}$ geborenenalter unter 28 Tagen [9].

Ein weiblicher Säugling im Alter von 2 Monaten erkrankte an einer Pneumonie [7], darüber hinaus zeigen 2 kleinere chinesische Fallserien [30, 35] bei Säuglingen vorwiegend das Vorliegen von Infektionszeichen der oberen Atemwege. Die große vergleichende Studie von Dong et al. belegt jedoch insgesamt ein erhöhtes Risiko für einen schwereren Verlauf von COVID-19 im Säuglingsalter [10].

\section{Kinder mit Vorerkrankungen}

Insgesamt wurde nur von 12 Kindern mit Vorerkrankungen in den vorgestellten Fallserien berichtet. In den beiden größten Studien sind die Angaben dazu allerdings nicht [10] oder nur unvollständig [21] vorhanden, sodass von einem „underreporting“ auszugehen ist. Auffällig war die häufige Nennung kardiologischer Vorerkrankungen (8 von 12 Patienten), die jedoch lediglich aus 2 Fallserien gemeldet wurde, sodass hier möglicherweise ein zentrumsbedingtes ,overreporting" besteht $[32,34]$.

Bisher gibt es erstaunlich wenige Meldungen $\mathrm{zu}$ immunkompromittierten Kindern mit COVID-19. Bei dem in den Studien von Lu et al. [21] und Sun et al. [28] sowie in dem Fallbericht von Chen et al. [6] berichteten 8-jährigen Jungen mit akuter lymphatischer Leukämie, chemotherapieassoziierter Granulozytopenie und COVID-19-assoziierter Pneumonie handelt es sich um denselben Patienten. Ein erster Bericht aus einem pädiatrischen Transplantationszentrum aus der Lombardei/Italien $\mathrm{zu}$ immunkompromittierten Patienten erwähnt 3 positiv auf SARS-CoV-2 getestete Kinder, die jedoch keine Komplikation oder klinische Zeichen einer Lungenerkrankung aufwiesen [8].

\section{Labor- und radiologische Diagnostik}

Im Blutbild waren bei bis zu zwei Dritteln der hospitalisierten Kinder keine Auffälligkeiten nachweisbar. Bei den übrigen
Kindern konnte kein eindeutiger Trend bezüglich der Erhöhung bzw. Erniedrigung der Leukozyten, Lymphozyten und Thrombozyten beobachtet werden. Auch bei schwer und kritisch erkrankten Kindern zeigte sich im Blutbild kein klarer Hinweis. Dies deckt sich mit der Übersichtsarbeit zu Laborwerten von 66 Kindern mit SARS-CoV-2-Infektion von Henry et al. [12]. Hingegen wurden in Studien bei Erwachsenen mit schweren Verläufen häufiger erhöhte Leukozytensowie erniedrigte Lymphozytenzahlen beschrieben $[5,18]$.

Deutliche Anstiege der LDH und der D-Dimere wurden in einzelnen Studien bei bis zu der Hälfte der Kinder mit schweren Verläufen [21, 28, 34] sowie bei dem verstorbenen Säugling [21] beobachtet. Analog dazu wurden Erhöhungen der D-Dimere in Erwachsenenstudien als Risikofaktor für schwere Verläufe identifiziert [5] und könnten als Hinweise auf thrombotische und ischämische Komplikationen im pulmonalen Gefäßbett interpretiert werden.

Bezüglich der bildgebenden Diagnostik ließen sich im CT des Thorax milchglasartige und fleckige Verschattungen als typische Zeichen der COVID19-Pneumonie frühzeitig nachweisen, teilweise auch bei asymptomatischen und PCR-negativen Kindern [23, 32]. Die auffälligen Befunde fanden sich vorwiegend in den Unterlappen sowie in den pleuranahen, peripheren Lungenabschnitten. Die Indikation zu einer CT-Untersuchung sollte wie bei anderen Erkrankungen sehr kritisch gestellt werden, da es keine eindeutige Korrelation zum klinischen Verlauf gibt und somit nicht notwendigerweise therapeutische Konsequenzen abgeleitet werden können. Stets sollte die hohe Strahlenbelastung der Untersuchung bei der Indikationsstellung bedacht werden [1].

\section{Vergleich COVID-19 zu anderen respiratorischen Viruserkrankungen}

Einige Autoren evaluierten auch den Schweregrad von COVID-19 bei Kindern im Vergleich zu anderen viralen Erkrankungen anhand von Literaturangaben. Klinisch scheint das deutlich 
seltenere Auftreten von Halsschmerzen bzw. Pharyngitis ein mögliches Unterscheidungsmerkmal im Vergleich zu Influenza-A-H1N1 zu sein (3\% vs. 95\%) [26]. Nach Qiu et al. [26] kommt es zwar bei Kindern mit COVID-19 häufiger $\mathrm{zu}$ einer Pneumonie als bei Kindern mit Influenza-A-H1N1 (53\% vs. 11\%), jedoch sei der Anteil an schweren Verläufen deutlich seltener ( $0 \%$ vs. $11 \%)$. Auffällig bei COVID-19-Patienten war in dieser Studie auch eine Erhöhung der myokardialen Enzyme ( $31 \%$ vs. $11 \%$ ) [26]. Gegenüber anderen epidemisch auftretenden Coronaviren wie SARS$\mathrm{CoV}$ und MERS-CoV scheint eine Infektion mit SARS-CoV-2 in der Regel deutlich weniger schwer $\mathrm{zu}$ verlaufen [26, 36].

\section{Limitationen}

Insgesamt waren die Fallserien von sehr heterogener Qualität. Angaben insbesondere zu Vorerkrankungen und Medikation sind nur teilweise vorhanden. Vor allem in der Anfangsphase der Epidemie ist davon auszugehen, dass möglicherweise ein Teil der Kinder primär zur Diagnostik/Isolierung und nicht aufgrund von COVID-19-Symptomen hospitalisiert wurde. Darauf weisen die Angabe von „asymptomatischen“ Kindern und der z. T. hohe Anteil an "milden“ Infektionen in den Fallserien hin. Die Nennung vermutlich z.T. derselben $\mathrm{Pa}$ tienten mit Vorerkrankung, Koinfektionen und kritischen Verläufen in verschiedenen Studien erschwert zudem die Einschätzung deren tatsächlicher Häufigkeit. Die z.T. auch mit antiviralen Medikamenten durchgeführten Therapieversuchelassen aufgrund fehlender Kontrollen keinen Rückschluss auf ihre Wirksamkeit zu.

\section{Ausblick}

Die dargestellten Daten aus Fallserien zu insgesamt 2518 Kindern mit COVID-19 aus China zeigen bei hospitalisierten Kindern einen in der Regel milderen Verlauf als bei Erwachsenen. Schwere und kritische Verläufe bei Kindern und Jugendlichen wurden bisher auch bei Risikogruppen nur selten berichtet, auch wenn Säug- linge und Kleinkinder hierfür ein insgesamt leicht erhöhtes Risiko aufweisen. Inwiefern eine Erhöhung der $\mathrm{LDH}$ und der D-Dimere oder andere Laborparameter bzw. Biomarker mit einem schwereren Verlauf assoziiert sind, muss weiter untersucht werden. Aufgrund der verfügbaren Daten erscheint eine Therapie mit antiviralen und/oder antiinflammatorischen Medikamenten derzeit allenfalls als Heilversuch in Ausnahmefällen indiziert. Weitere möglichst populationsbezogene Daten sowie Daten zu Risikopatienten werden dringend benötigt, um das neue Krankheitsbild COVID-19 bei Kindern einordnen und mögliche präventive wie therapeutische Maßnahmen möglichst effizient und sicher anwenden zu können.

\section{Fazit für die Praxis}

- In der Pädiatrie ist von einer hohen Anzahl von oligosymptomatischen und asymptomatischen Kindern mit SARS-CoV-2-Infektion auszugehen.

- Bei hospitalisierten Kindern mit COVID-19 überwiegen leichte bis moderate Verläufe, oft als leichte Pneumonie. Todesfälle sind sehr selten.

- Säuglinge und Kleinkinder sind häufiger von COVID-19-Hospitalisierungen betroffen. Vereinzelt werden schwere Verläufe berichtet.

- Die Datenlage zu Risikogruppen, Laborparametern und Therapie ist bislang begrenzt.

- Weitere Erhebungen sind dringend erforderlich, insbesondere ergibt sich über die Beteiligung aller Kinderkliniken am DGPI COVID-19-Survey die Möglichkeit, innerhalb kurzer Zeit die in Deutschland verfügbaren Daten zu erfassen und zugänglich zu machen (https://dgpi.de/).

\section{Korrespondenzadresse}

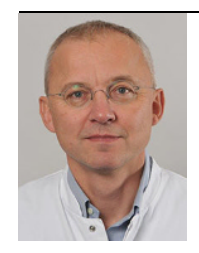

Prof. Dr. med. J. G. Liese, MSc

Kinderklinik und Poliklinik, Universitätsklinikum an der Julius-MaximiliansUniversität Würzburg Würzburg, Deutschland Liese_J@ukw.de
Einhaltung ethischer Richtlinien

Interessenkonflikt. A. Streng, K. Hartmann, J. Armann, R. Berner und J. Liese geben an, dass kein Interessenkonflikt besteht.

Für diesen Beitrag wurden von den Autoren keine Studien an Menschen oder Tieren durchgeführt. Für die aufgeführten Studien gelten die jeweils dort angegebenen ethischen Richtlinien.

\section{Literatur}

1. Antoch G, Urbach H, Mentzel HJ (2020) SARS-CoV2/COVID-19: Empfehlungen für die Radiologische Versorgung. Fortschr Röntgenstr. https://doi.org/ 10.1055/a-1149-3625

2. Cai J, Xu L, Lin D et al (2020) A case series of children with 2019 novel coronavirus infection: clinical and epidemiological features. Clin Infect Dis. https:// doi.org/10.1093/cid/ciaa198

3. Cao Q, Chen Y-C, Chen C-L et al (2020) SARSCoV-2 infection in children: transmission dynamics and clinical characteristics. J Formos Med Assoc 119:670-673

4. Chan JF, Yuan S, Kok KH et al (2020) A familial cluster of pneumonia associated with the 2019 novel coronavirus indicating person-to-person transmission: a study of a family cluster. Lancet 395:514-523

5. Chen T, Wu D, Chen H et al (2020) Clinical characteristics of 113 deceased patients with coronavirus disease 2019: retrospective study. BMJ 368:m1091

6. Chen Z, Xiong H, Li JX et al (2020) COVID-19 with post-chemotherapy agranulocytosis in childhood acute leukemia: a case report. Zhonghua Xue Ye Xue Za Zhi 41(0):E4

7. Cui Y, Tian Mm Huang D et al (2020) A 55-day-old female infant infected with COVID 19: presenting with pneumonia, liver injury, and heart damage. JInfect Dis. https://doi.org/10.1093/infdis/jiaa113

8. D'Antiga L (2020) Coronaviruses and immunosuppressed patients. The facts during the third epidemic. Liver Transpl. https://doi.org/10.1002/ LT.25756

9. DGPIhttps://dgpi.de.Zugegriffen:6. Apr. 2020

10. Dong Y, Mo X, Hu Y et al (2020) Epidemiological characteristics of 2143 pediatric patients with 2019 coronavirus disease in China. Pediatrics. https:// doi.org/10.1542/peds.2020-0702

11. ECDC http://www.ecdc.europa.eu/en. Zugegriffen:3. Apr. 2020

12. Henry BM, Lippi G, Plebani M (2020) Laboratory abnormalities in children with novel coronavirus disease 2019. Clin Chem Lab Med. https://doi.org/ 10.1515/cclm-2020-0272

13. Illinois Department of Public Health (2020) Public health officials announce the first death of aninfant with coronavirus disease. http://dph.illinois.gov/ news/public-health-officials-announce-firstdeath-infant-coronavirus-disease. Zugegriffen: 5. Apr. 2020

14. IstitutoSuperiore diSanità https://www.epicentro. iss.it/coronavirus/bollettino/Infografica_3aprile \%20ENG.pdf.Zugegriffen:4. Apr. 2020

15. Kamali Aghdam M, Jafari N, Eftekhari K (2020) Novel coronavirus in a 15-day-old neonate with clinical signs of sepsis, a case report. Infect Dis (Lond) 1:1-3 
16. Karimi-Zarchi M, Neamatzadeh H, Dastgheib SA et al (2020) Vertical transmission of coronavirus disease 19 (COVID-19) from infected pregnant mothers to neonates: a review. Fetal Pediatr Pathol 2:1-5

17. Li Y, Zhao R, Zheng S et al (2020) Lack of vertical transmission of severe acute respiratory syndrome coronavirus 2, China. Emerg Infect Dis. https://doi. org/10.3201/eid2606.200287

18. Lippi G, Plebani M (2020) Laboratory abnormalities in patients with COVID-2019 infection. Clin Chem Lab Med. https://doi.org/10.1515/cclm-20200198

19. Liu W, Zhang Q, Chen J et al (2020) Detection of COVID-19 in children in early January 2020 in Wuhan, China. N Engl J Med. https://doi.org/10. 1056/NEJMc2003717

20. Lu Q, Shi Y (2020) Coronavirus disease (COVID-19) and neonate: What neonatologists need to know. JMed Virol. https://doi.org/10.1002/jmv.25740

21. Lu X, Zhang L, Du H et al (2020) SARS-CoV-2 infection in children. N Engl J Med. https://doi.org/ 10.1056/NEJMc2005073

22. Ludvigsson JF (2020) Systematic review of COVID19 in children show milder cases and a better prognosis than adults. Acta Paediatr. https://doi. org/10.1111/APA.15270

23. $\mathrm{Ma} \mathrm{H}, \mathrm{Hu}$ J, Tian J et al (2020) Visualizing the novel coronavirus (COVID-19) in children: what we learn from patients at Wuhan children's hospital. SSRN Journal. https://doi.org/10.2139/ssrn.3550012

24. Ministerio De Sanidad Gobierno de España (2020) Actualización n65. Enfermedad por el coronavirus (COVID-19). 04.04.2020 (datos consolidados a las 21:00 horas del 03.04.2020). https:// www.mscbs.gob.es/profesionales/saludPublica/ ccayes/alertasActual/nCov-China/documentos/ Actualizacion_65_COVID-19.pdf. Zugegriffen: 5. Apr. 2020

25. Mullins E, Evans D, Viner RN et al (2020) Coronavirus in pregnancy and delivery: rapid review. Ultrasound Obstet Gynecol. https://doi. org/10.1002/uog.22014

26. Qiu H, Wu J, Hong L et al (2020) Clinical and epidemiological features of 36 children with coronaviorus disease 2019 (COVID-19) in Zhejiang, China: an observational cohort study. Lancet Infect Dis. https://doi.org/10.1016/S14733099(20)30198-5

27. Robert Koch-Institut (2020) Täglicher Lagebericht des RKI zur Coronavirus-Krankheit-2019 (COVID-19). https://www.rki.de/DE/Content/InfAZ/ N/Neuartiges_Coronavirus/Situationsberichte/ 2020-04-02-de.pdf?_blob=publicationFile. Zugegriffen: 5. Apr. 2020

28. Sun D, Li H, Lu XX et al (2020) Clinical features of severe pediatric patients with coronavirus disease 2019 in Wuhan: a single center's observational study. World J Pediatr. https://doi.org/10.1007/ s12519-020-00354-4

29. Wang D, Ju XL, Xie F et al (2020) Clinical analysis of 31 cases of 2019 novel coronavirus infection in children from six provinces [autonomous region] of northern China.Zhonghua Er KeZaZhi 58(4):E11

30. Wei M, Yuan J, Liu Y et al (2020) Novel coronavirus infection in hospitalized infants under 1 year of age in China. JAMA. https://doi.org/10.1001/jama. 2020.213

31. Wu Z, McGoogan JM (2020) Characteristics of and important lessons from the coronavirus disease 2019 (COVID-19) outbreak in China: summary of a report of 72314 cases from the Chinese center for disease control and prevention. JAMA. https://doi. org/10.1001/jama.2020.2648
32. Xia W, Shao J, Guo Y et al (2020) Clinical and CT features in pediatric patients with COVID-19 infection: different points from adults. Pediatr Pulmonol. https://doi.org/10.1002/ppul.24718

33. Zeng LK, Tao XW, Yuan WH et al (2020) First case of neonate infected with novel coronavirus pneumonia in China. Zhonghua Er Ke Za Zhi. https://doi.org/10.3760/cma.j.issn.0578-1310. 2020.0009

34. Zheng F, Liao C, Fan QH et al (2020) Clinical characteristics of children with coronavirus disease 2019 in Hubei, China. Curr Med Sci 40(2):1-6. https://doi.org/10.1007/s11596-020-2172-6

35. ZhouY, Yang GD, Feng Ketal (2020)Clinical features and chest CT findings of coronavirus disease 2019 in infants and young children. Zhongguo Dang Dai ErKeZaZhi 22(3):215-220

36. Zimmermann P, Curtis N (2020) Coronavirus infections in children including COVID-19. An overview of the epidemiology, clinical features, diagnosis, treatment and prevention options in children. Pediatr Infect Dis J. https://doi.org/10. 1097/INF.0000000000002660
Typ-1-Diabetes und Zöliakie treten häufig im Duo auf

Während weniger als $0,5 \%$ der Normalbevölkerung von Zöliakie betroffen sind, ist die Zahl bei Menschen mit Typ-1-Diabetes aufgrund eines identischen genetischen Hintergrunds bedeutend höher. Die Häufigkeit für Zöliakie bei Kindern und Jugendlichen mit Typ-1-Diabetes liegt bei 3-6\%, wobei die Zahlen international schwanken. Neben regionalen Unterschieden im Zöliakie-Risiko spiegeln diese Schwankungen auch unterschiedliche Screeningbeziehungsweise Diagnostik-Praktiken wider. "Leider werden noch nicht in allen Kliniken Kinder und Jugendliche mit einem frisch manifestierten Typ-1-Diabetes standardmäßig auf Zöliakie-Antikörper untersucht, obwohl dies in der S3-Leitlinie der Deutschen Diabetes Gesellschaft und der Arbeitsgemeinschaft für Pädiatrische Diabetologie empfohlen wird", sagt Professor Dr. med. Thomas Danne, Chefarzt am Kinderkrankenhaus Auf der Bult in Hannover und Mitglied im wissenschaftlichen Beirat von diabetesDE - Deutsche Diabetes-Hilfe. Daher gebe es auch viele Betroffene, bei denen die Zöliakie später oder gar erst im Erwachsenenalter festgestellt werde.

Durch die Zöliakie kommt es bei Betroffenen unter anderem zu Verdauungsbeschwerden, Stoffwechselschwankungen und auch zu Mangelerscheinungen. „Bei einem positiven Antikörperbefund sollte bei Kindern mit Typ-1-Diabetes spätestens mit 8 Jahren auch die feingewebliche Untersuchung einer Dünndarmgewebeprobe erfolgen", erläutert Professor Danne. Sonst kann aufgrund einer unerkannten Zöliakie auch eine Osteoporose entstehen, die im pubertären Wachstumsschub zu Problemen führt.

Menschen mit Zöliakie müssen lebenslang eine glutenfreie Ernährung einhalten. Glutenfreie Produkte können sich jedoch anders auf den Blutzuckerspiegel auswirken als entsprechende glutenhaltige Lebensmittel, betont der Kinderdiabetologe: „Betroffene Menschen mit Typ-1-Diabetes müssen daher ihre Berechnung der Kohlenhydrate und die benötigte Insulinmenge an diese neue Situation anpassen."

Quelle: www.deutsche-diabeteshilfe.de 
Hier steht eine Anzeige.

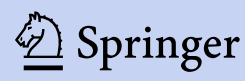

